\title{
Access Management and Control using NFC
}

\author{
Swastika Shukla ${ }^{1}$, Prachi Shroff ${ }^{2}$, Vivek Nair ${ }^{3}$, Rijo Kuruvilla ${ }^{4}$ \\ 1, 2, 3, 4 Xavier Institute of Engineering, Mumbai University, Mahim Causeway, Opp Raheja Hospital, Mumbai, Maharashtra 400016, India
}

\begin{abstract}
Near Field Communication (NFC) has a huge potential to simplify our everyday tasks, ranging from paying for items to accessing our office or home. In this context we will focus on using NFC together with a arduino circuit board and NFC reader anad tags to realize a simple system for granting access to open a locked door.
\end{abstract}

Keywords: NFC reader, NFC tags, Arduino UNO, door lock

\section{Introduction}

Recently, lot of researches have been devoted to a technology-based home security and automation. As there is rapid development in the fields of communication/networks and other related wireless technologies such as RFID (Radio Frequency Identification),UWB (Ultra Wide Band), Zigbee, NFC (Near Field Communication) and Bluetooth, these enable us to develop various kinds of wireless systems via handsets or smartphones. NFC is defined as a short range wireless communication protocol that is primarily intended to be used on smartphone. It operates based on a technology named as Radio Frequency Identification (RFID) technology, and it is a contactless/wireless system that uses radio frequency (RF) waves to transferring data to or from a short distance from a tag, approximately several centimetres away [2]. Bluetooth and Wi-Fi seem similar to the near field communication technology as all three allow wireless communication and data exchange between digital devices which support Near field communication. However, Bluetooth and Wi-Fi focus on radio transmissions and near field communication utilizes the electromagnetic radio fields [4]. Research use handset and actuator for remote operation of various electrical devices at home. Keyless entry is just another way we can use to make our lives easier, convenient but also secure.

The aim is to create easy-to-make keyless entry system which senses when there is an NFC tag present and unlocks your door.The main goal of this project is to develop a NFC door lock system where an administrator or owner of an apartment or building can unlock the door without using conventional keys, in a way which is effortless and not at all time consuming. Thus the person can manage and send "digital keys" to other persons in order to allow them to access an apartment, building, or specific room in a building by using their NFC smartcard.

In order to realize NFC Door Lock is a system we need to achieve these goals:

- Connect the Arduino UNO circuit board to a network,

- Configure the NFC reader,

- Connect the NFC reader to the Arduino board,

- Write Arduino code for authenticating users,

- Connect sensors (leds and buzzer) to indicate the access provided.

In this project, the door lock will consist of an NFC reader which will authenticate the user. The user has an NFC card which will be programmed in such that when it is taken near the door lock i.e. at the distance of approximately 10 meters, an indication that the user is authorized will be generated. It could be an green led or a beep or any other sound and if it is not authorized then a red led or an error would be generated. The door would open if authenticated and would stay open for a précised time to enter the room else it would not open. And this is how we will Implement NFC in door lock.

One of the purposes of this realization is to explore what services can be realized when such a system is connected to the home/building network. We hope that the end result of this project will lead to a simpler life by exploiting this increasingly ubiquitous technology.

\section{Literature Survey}

In today's fast -growing technology world, most of mobile devices are equipped with wireless modules, which can be used to solve the problems which we face with keys. Almost all these technologies are equipped with Bluetooth and infrared, among which the most recent technology is NFC. Compared to other short-range technologies, NFC has the following advantages:

- Slow speed and short range - this allows NFC to consume as little power as possible so it can be left on at all times and not affect the phone's battery by that much (vs. Bluetooth);

- Hassle-free approach to connections - with NFC, bringing the two devices within range is enough to facilitate the communication between the two (vs. Bluetooth);

- Free-line of sight - no direct line of sight is required to establishes connection (vs. Infrared)[3]

Each full NFC devices can work in three modes:

- NFC card emulation: This enables NFC-enabled devices such as smartphones to act like a key, in order to allow users to perform transactions such as payment or ticketing.

- NFC reader/writer: The information stored on inexpensive NFC tags embedded in labels or smart posters are read by these NFC readers.

- NFC peer-to-peer: Two NFC-enabled devices communicate with each other to exchange information in an adhoc fashion [1]. 


\section{International Journal of Science and Research (IJSR) \\ ISSN (Online): 2319-7064}

Index Copernicus Value (2013): 6.14 | Impact Factor (2014): 5.611

NFC has many applications like data transformation from mobile-to-mobile, mobile-to laptop, authentication and access control, etc. We are using NFC for access control and authentication. We are implementing NFC technology on door locks for access control and authentication. In this we are using NFC readers, NFC tags and arduino as the main components.

There were some researches done in previous years for implementing the attendance control systems in universities. Authors of research work used RFID-technology as an automatic monitor of student classroom attendance. They demonstrated how to automate an entire student-attendance registration system within an educational institution by the use of Ethernet. However, there was some other research work done with different views for attendance checking system. Authors designed and implemented wireless iris recognition attendance management system, whereas in authors proposed attendance management system extended with computer vision algorithms. And finally, authors implemented a system for attendance checking based in RFID-technology [3]. In most of this research work, RFIDtechnology was used a framework for building systems, whereas authors of this research paper presents an NFCenabled Access Control System, which by the help of NFC technology makes possible for people to use only one single key.

\section{Problem Definition}

Near field communication, abbreviated NFC, is a form of contactless communication between devices like smartphones or tablets or any devices which support NFC[4].Nowadays, the number of problems with conventional keys such as building break-in cases are increasing and these problem are increasingly severe from time to time. An access control system which is proposed serves as necessity prevention in order to reduce the number of building break-in and other problems, also at the same time, it provides a safer alternative in security perspective. An access control system is simply defined as any technique used to control passage into or out of any area or any entry, such as residential area, office and others. The evolution of science and technology creates a new generation of the access control system, known as digital access control system [2].

\section{Methodology}

\subsection{Circuit diagram}

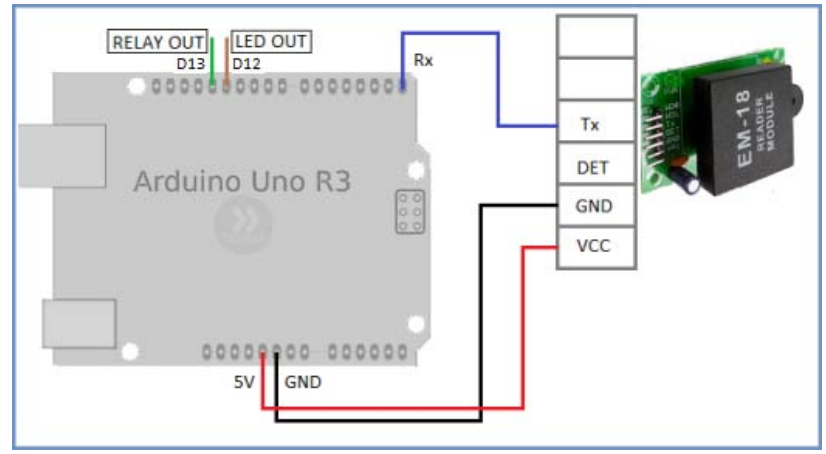

Figure 1: Circuit diagram
Arduino Uno an open source physical computing platform based on ATmega328 microcontroller and provides a development environment for writing software for the board. It can be used for a variety of projects.

EM-18 RFID reader is one of the commonly used RFID readers to read $125 \mathrm{KHz}$ tags. It features low cost, low power consumption, small form factor and easy to use. It provides both UART and Wiegand26 output formats. It can be directly interfaced with microcontrollers using UART and with PC using an RS232 converter. The module radiates $125 \mathrm{KHz}$ through its coils and when a $125 \mathrm{KHz}$ passive RFID tag is brought into this field it will get energized from this field. These passive RFID tags mostly consist of CMOS IC EM4102 which can get enough power for its working from the field generated by the reader. Interface an EM-18 RFID Reader with AVR ATmega16 microcontroller. Here, we will use the serial interface of EM-18 RFID Reader to interface the reader with the USART of microcontroller in asynchronous mode. When the RFID reader reads any RFID tag, the ATmega16 microcontroller will read the 12 byte data transmitted from RFID reader and it will indicate whether the user is allowed access by means of LED and buzzer and the door will be unlocked if valid tag is used else it will remain locked.

\subsection{Working}

In our project we will use RFID model EM 18, a tag and an arduino. NFC tags are passive data stores which can be read, and under some circumstances written to, by an NFC device. They typically contain data (as of 2015 between 96 and 8,192 bytes) and are read-only in normal use, but may be rewritable. Applications include secure personal data storage (e.g. debit or credit card information, loyalty program data, Personal Identification Numbers (PINs), contacts)[1]. Every tag consists of a unique tag number. When the tag is brought near to the reader tag number will be read provided RFID reader is connected to computer. This reading of UID i.e. tag no. is done using a software "Hiperterminal". The details of the tag will then be copied and saved for programing use. Now the Arduino will be connected to the computer and a program for working of LED and the door lock will be burnt on the arduino using the arduino software. A time limit for which the door would be open when user is authenticated is set. 


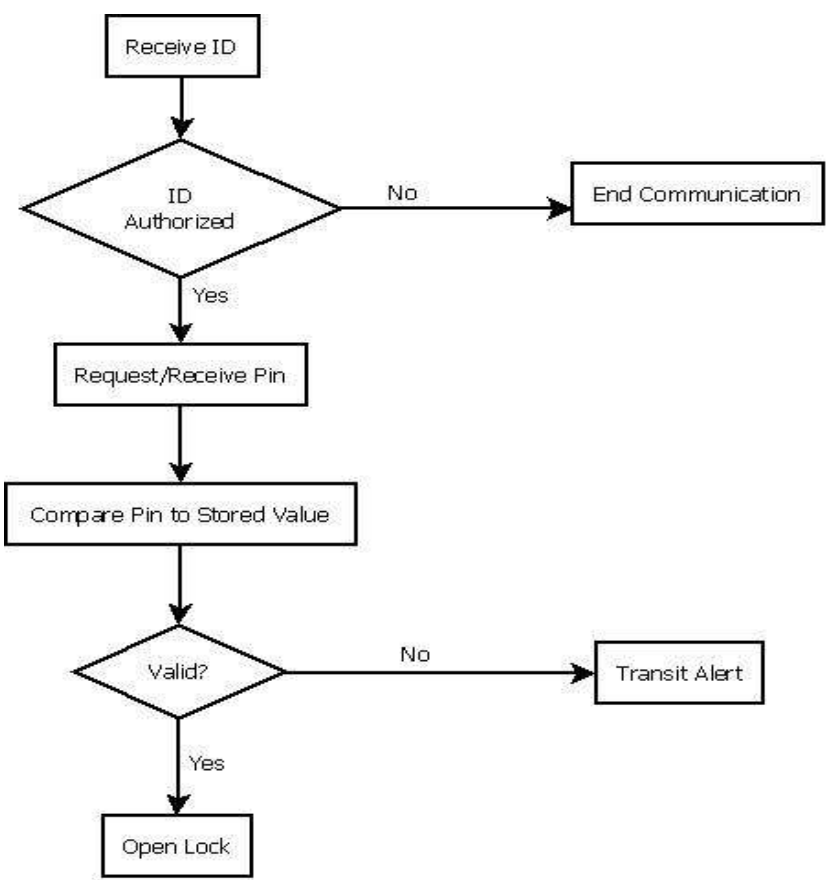

Figure 2: Flowchart for authentication process

\section{Conclusion and Future scope}

The proposed system will help in making the access system time efficient and effortless. NFC is becoming one of the most important advances in wireless communications technologies hence using this future technology will lead to a evolved and efficient system. In the future, we plan to make the system better in safety aspects, including different tags for registering a new tag or removing an already existing tag from the system and different tags for accessing to make the system perform more.

\section{References}

[1] Near field communication. [Online]. Available: https://en.wikipedia.org/wiki/Near_field_communicatio n. [Accessed: Nov.12, 2015]. (General Internet site)

[2] Peng-Loon Teh, Huo-Chong Ling, Soon-Nyean Cheong, "NFC Smartphone Based Access Control System U sing Information Hiding," 2013 IEEE Conference on Open Systems (ICOS), December 2 - 4, 2013, Sarawak, Malaysia. (conference style)

[3] Nurbek Saparkhojayev, Aybek Nurtayev and Gulnaz Baimenshina, "Access Control and Management System Based on NFC-Technology by the Use of Smart Phones as Keys," Middle-East Journal of Scientific Research 21 (7): 1130-1135, 2014. (journal style)

[4] Near field communication. [Online]. Available: http://www.nearfieldcommunication.org. [Accessed: Nov.12, 2015]. (General Internet site)

\section{Author Profile}

Swastika Shukla is pursuing her Bachelors Engineering degree in Computer Engineering. She is currently studying final year in Xavier Institute of Engineering, Mahim Causeway, Mahim, Mumbai, Maharashtra, India.

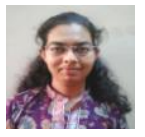

Prachi Shroff is pursuing her Bachelors Engineering degree in Computer Engineering. She is currently studying final year in Xavier Institute of Engineering, India. Mahim Causeway, Mahim, Mumbai, Maharashtra,

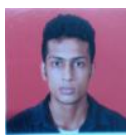

Vivek Nair is pursuing his Bachelors Engineering degree in Computer Engineering. He is currently studying final year in Xavier Institute of Engineering, Mahim Causeway, Mahim, Mumbai, Maharashtra, India.

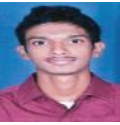

Rijo Kuruvilla is pursuing his Bachelors Engineering degree in Computer Engineering. $\mathrm{He}$ is currently studying final year in Xavier Institute of Engineering, India. Mahim Causeway, Mahim, Mumbai, Maharashtra, 